Canad. Math. Bull. Vol. 21 (3), 1978

\title{
THE HAUSDORFF MOMENT PROBLEM
}

\author{
BY \\ DAVID BORWEIN
}

1. Introduction. Suppose throughout that

$$
0 \leq \lambda_{0}<\cdots<\lambda_{n}, \quad \lambda_{n} \rightarrow \infty, \quad \sum_{n=1}^{\infty} \frac{1}{\lambda_{n}}=\infty,
$$

and that $\left\{\mu_{n}\right\}(n \geq 0)$ is a sequence of real numbers. The (generalized) Hausdorff moment problem is to determine necessary and sufficient conditions for there to be a function $x$ in some specified class satisfying

$$
\mu_{n}=\int_{0}^{1} t^{\lambda_{n}} d x(t) \text { for } n=0,1,2, \ldots
$$

Let

$$
D_{0}=d_{0}=1, \quad D_{n}=d_{0}+d_{1}+\cdots+d_{n}=\left(1+\frac{1}{\lambda_{1}}\right) \cdots\left(1+\frac{1}{\lambda_{n}}\right) .
$$

Define the divided difference $\left[\mu_{k}, \ldots, \mu_{n}\right]$ inductively by $\left[\mu_{k}\right]=\mu_{k}$,

$$
\left[\mu_{k}, \ldots, \mu_{n}\right]=\frac{\left[\mu_{k}, \ldots, \mu_{n-1}\right]-\left[\mu_{k+1}, \ldots, \mu_{n}\right]}{\lambda_{n}-\lambda_{k}} \text { for } 0 \leq k<n .
$$

For $0 \leq k \leq n, 0 \leq t \leq 1$, let

$$
\begin{aligned}
\lambda_{n k} & =\lambda_{k+1} \cdots \lambda_{n}\left[\mu_{k}, \ldots, \mu_{n}\right], \\
\lambda_{n k}(t) & =\lambda_{k+1} \cdots \lambda_{n}\left[t^{\lambda_{k}}, \ldots, t^{\lambda_{n}}\right]
\end{aligned}
$$

with the convention that products such as $\lambda_{k+1} \cdots \lambda_{n}=1$ when $k=n$. Let

$$
\begin{aligned}
& M_{p n}= \begin{cases}\left(\sum_{k=0}^{n}\left|\lambda_{n k}\right|^{p}\left(\frac{D_{n}}{d_{k}}\right)^{p-1}\right)^{1 / p} & \text { if } 1 \leq p<\infty, \\
\max _{0 \leq k \leq n}\left|\lambda_{n k}\right| \frac{D_{n}}{d_{k}} & \text { if } p=\infty ;\end{cases} \\
& M_{p}=\sup _{n \geq 0} M_{p n} .
\end{aligned}
$$

Received by the editors March 29, 1978.

This paper is one of a series of survey papers written at the invitation of the Editors of the Canadian Mathematical Bulletin.

This research was supported in part by the National Research Council of Canada, Grant A-2983. 
Let $C$ be the normed linear space of functons $x$ continuous on $[0,1]$ with norm $\|x\|_{C}=\sup _{0 \leq t \leq 1}|x(t)|$. Let $B V$ be the space of functions of bounded variation on $[0,1]$. A function $x \in B V$ is said to be normalized if $x(0)=0$ and $2 x(t)=x(t+)+x(t-)$ for $0<t<1$. For $p \geq 1$, let $L_{p}$ be the normed linear space of measurable functions $x$ on $(0,1)$ with finite norm $\|x\|_{p}$ where

$$
\|x\|_{p}=\left\{\begin{array}{lll}
\left(\int_{0}^{1}|x(t)|^{p} d t\right)^{1 / p} & \text { when } & 1 \leq p<\infty \\
\underset{0<t<1}{\text { ess. sup }} & \text { when } p=\infty .
\end{array}\right.
$$

It is known that $M_{1}<\infty$ if and only if there is a functon $\alpha \in B V$ satisfying

$$
\mu_{n}=\int_{0}^{1} t^{\lambda_{n}} d \alpha(t) \text { for } n=0,1,2, \ldots
$$

The case $\lambda_{0}=0$ of this result was established by Hausdorff [5], [6] and Schoenberg [12] subsequently gave a different proof. The case $\lambda_{0}>0$ was proved by Leviatan [9] (see also Endl [4]).

It can be deduced from theorems of Leviatan [9, Theorem 2.3; 10, Theorem 1 and Theorem 2] (see also Berman [3]) and identity (5) (below) that, for $1<p \leq \infty, M_{p}<\infty$ if and only if there is a function $\beta \in L_{p}$ satisfying

$$
\mu_{n}=\int_{0}^{1} t^{\lambda_{n}} \beta(t) d t \quad \text { for } \quad n=0,1,2, \ldots
$$

The case $\lambda_{n}=n$ for $n=0,1,2, \ldots$ of this result is due to Hausdorff [7]. In this case we have that for $0 \leq k \leq n, 0 \leq t \leq 1$,

$$
\lambda_{n k}(t)=\left(\begin{array}{l}
n \\
k
\end{array}\right) t^{k}(1-t)^{n-k}, \quad \lambda_{n k}=\left(\begin{array}{l}
n \\
k
\end{array}\right) \Delta^{n-k} \mu_{k}
$$

where $\Delta^{0} \mu_{k}=\mu_{k}, \Delta^{n} \mu_{k}=\Delta^{n-1} \mu_{k}-\Delta^{n-1} \mu_{k+1}$.

In this paper we give new and reasonably self-contained proofs of the above results. Our proofs involve functional analysis and differ radically from those of the above-mentioned authors. Unlike previous proofs, ours do not treat the cases $\lambda_{0}=0$ and $\lambda_{0}>0$ separately.

In addition, we show that if (1) holds with $\alpha$ normalized, then $M_{1}=\int_{0}^{1}|d \alpha(t)|$ when $\lambda_{0}=0$, and $M_{1}=\int_{0}^{1}|d \alpha(t)|-|\alpha(0+)|$ when $\lambda_{0}>0$. We also show that if (2) holds for $1<p \leq \infty$, then $M_{p}=\|\beta\|_{p}$. Finally, we show that $M_{p n}$ increases with $n$ and hence that $M_{p}=\lim _{n \rightarrow \infty} M_{p n}$ for $1 \leq p \leq \infty$. The cases $\lambda_{n}=n$ for $n=$ $0,1,2, \ldots$ of these results are derived in a book by Shohat and Tamarkin [13, pp. 97-101]. This book, incidentally, gives an excellent and extensive review of the classical moment problem. Another good reference book on the subject is one by Akhiezer [1]. 
2. Preliminary results. The following simple identities and inequalities are known:

$$
\begin{gathered}
\mu_{s}=\sum_{k=0}^{n} \lambda_{n k}\left(1-\frac{\lambda_{s}}{\lambda_{k+1}}\right) \cdots\left(1-\frac{\lambda_{s}}{\lambda_{n}}\right) \text { for } 0 \leq s \leq n . \\
0 \leq \lambda_{n s}(t) \leq \sum_{k=0}^{n} \lambda_{n k}(t) \leq 1 \text { for } 0 \leq t \leq 1, \quad 0 \leq s \leq n .
\end{gathered}
$$

$$
\int_{0}^{1} \lambda_{n k}(t) d t=\frac{d_{k}}{D_{n}} \text { for } 0 \leq k \leq n .
$$

We require some lemmas.

LemMa 1. If $M_{1}<\infty$, then

$$
\mu_{s}=\lim _{n \rightarrow \infty} \sum_{k=0}^{n} \lambda_{n k}\left(\frac{D_{k}}{D_{n}}\right)^{\lambda_{s}} \text { for } s=0,1,2, \ldots
$$

Proof. Let $\lambda>0, u_{n}=e^{-\lambda / \lambda_{n}}$,

$$
\phi_{n}(\lambda)=\sum_{k=0}^{n} \lambda_{n k} u_{k+1} \cdots u_{n}
$$

and let

$$
\psi_{n}=\sum_{k=0}^{n} \lambda_{n k} v_{k+1} \cdots v_{n}
$$

where $v_{n}=e^{-\gamma_{n} / \lambda_{n}}$ for sufficiently large $n$ and $\gamma_{n} \rightarrow \lambda$ as $n \rightarrow \infty$.

Let $0<\varepsilon<\lambda$. Then, for $\delta>0,|\gamma-\lambda|<\varepsilon$, we have that

$$
\left|e^{-\delta \lambda}-e^{-\delta \gamma}\right| \leq \delta|\gamma-\lambda| e^{-\delta(\lambda-\varepsilon)} \leq \frac{\varepsilon}{\lambda-\varepsilon} .
$$

Choose a positive integer $N$ so large that $\left|\gamma_{n}-\lambda\right|<\varepsilon$ for $n>N$. Then, for $n>N$, we have that

$$
\left|\psi_{n}-\phi_{n}(\lambda)\right| \leq M_{1} \sum_{k=0}^{N-1}\left|v_{k+1} \cdots v_{n}-u_{k+1} \cdots u_{n}\right|+\frac{\varepsilon}{\lambda-\varepsilon} \sum_{k=N}^{n}\left|\lambda_{n k}\right| .
$$

Since $u_{n} \rightarrow 0$ and $v_{n} \rightarrow 0$ as $n \rightarrow \infty$, it follows that

$$
\limsup _{n \rightarrow \infty}\left|\psi_{n}-\phi_{n}(\lambda)\right| \leq \frac{M_{1} \varepsilon}{\lambda-\varepsilon},
$$

and hence that

$$
\lim _{n \rightarrow \infty}\left(\psi_{n}-\phi_{n}(\lambda)\right)=0
$$


Note that when $v_{n}=1-\lambda_{s} / \lambda_{n}$, then, by (3), the corresponding $\psi_{n}=\mu_{s}$ for $n \geq s$. Thus

$$
\lim _{n \rightarrow \infty} \phi_{n}\left(\lambda_{s}\right)=\mu_{s} .
$$

The desired conclusion is now obtained by considering the $\psi_{n}$ corresponding to

$$
v_{n}=\left(1+\frac{1}{\lambda_{n}}\right)^{-\lambda_{s}} \text {. }
$$

\section{LEMMA 2.}

(i) If (1) is satisfied by a function $\alpha \in B V$, then $M_{1} \leq \int_{0}^{1}|d \alpha(t)|$.

(ii) If $1<p \leq \infty$ and (2) is satisfied by a function $\beta \in L_{p}$, then $M_{p} \leq\|\beta\|_{p}$.

Proof. Part (i). We have that

$$
\lambda_{n k}=\int_{0}^{1} \lambda_{n k}(t) d \alpha(t) \text { for } 0 \leq k \leq n,
$$

and thus, by (4), that

$$
\sum_{k=0}^{n}\left|\lambda_{n k}\right| \leq \int_{0}^{1}|d \alpha(t)| \sum_{k=0}^{n} \lambda_{n k}(t) \leq \int_{0}^{1}|d \alpha(t)| .
$$

Hence

$$
M_{1} \leq \int_{0}^{1}|d \alpha(t)| .
$$

Part (ii). We now have that

$$
\lambda_{n k}=\int_{0}^{1} \lambda_{n k}(t) \beta(t) d t \text { for } 0 \leq k \leq n .
$$

Hence, by (5),

$$
\left|\lambda_{n k}\right| \leq \int_{0}^{1} \lambda_{n k}(t)|\beta(t)| d t \leq \frac{d_{k}}{D_{n}} \underset{0<t<1}{\operatorname{ess} . \sup }|\beta(t)| .
$$

Next, if $1<p<\infty$, then, by Hölder's inequality and (5),

$$
\begin{aligned}
\left|\lambda_{n k}\right|^{p} & \leq \int_{0}^{1} \lambda_{n k}(t)|\beta(t)|^{p} d t\left(\int_{0}^{1} \lambda_{n k}(t) d t\right)^{p-1} \\
& =\left(\frac{d_{k}}{D_{n}}\right)^{p-1} \int_{0}^{1} \lambda_{n k}(t)|\beta(t)|^{p} d t ;
\end{aligned}
$$

and so, by (4),

$$
\sum_{k=0}^{n}\left|\lambda_{n k}\right|^{p}\left(\frac{D_{n}}{d_{k}}\right)^{p-1} \leq \int_{0}^{1}|\beta(t)|^{p} d t \sum_{k=0}^{n} \lambda_{n k}(t) \leq \int_{0}^{1}|\beta(t)|^{p} d t .
$$

Consequently, if $1<p \leq \infty$, then $M_{p} \leq\|\beta\|_{p}$. 
LEMMA 3. If a normalized function $x \in B V$ is such that

$$
\int_{0}^{1} t^{\lambda_{n}} d x(t)=0 \text { for } n=0,1,2, \ldots,
$$

then $x(t)=x(0+)$ for $0<t \leq 1$. If, in addition, $\lambda_{0}=0$, then $x(0+)=0$.

Proof. Suppose first that $\lambda_{0}=0$. A known consequence of the hypothesis $[11$, p. 337] is that

$$
\int_{0}^{1} t^{n} d x(t)=0 \text { for } n=0,1,2, \ldots
$$

Hence, by a standard result [14, Theorem 6.1], $x(t)=0$ for $0 \leq t \leq 1$.

Suppose next that $\lambda_{0}>0$. Then, by hypothesis,

$$
\int_{0}^{1} t^{\lambda_{n}-\lambda_{0}} d y(t)=0 \text { for } n=0,1,2, \ldots,
$$

where $y(t)=\int_{0}^{t} u^{\lambda_{0}} d x(u)$. Since $y$ is normalized [14, Theorem 8b], we have, by the part already proved, that $y(t)=0$ for $0 \leq t \leq 1$. Let $0<\varepsilon \leq t \leq 1$. Then

$$
0=\int_{\varepsilon}^{t} u^{\lambda_{0}} d x(u)=t^{\lambda_{0}} x(t)-\varepsilon^{\lambda_{0}} x(\varepsilon)=\int_{\varepsilon}^{t} u^{\lambda_{0}-1} x(u) d u
$$

and so $x$ is absolutely continuous in $[\varepsilon, 1]$. Therefore $0=\int_{\varepsilon}^{t} u^{\lambda_{0}} x^{\prime}(u) d u$ and consequently $x^{\prime}(u)=0$ a.e. in $(\varepsilon, 1)$. It follows that $x(t)=x(\varepsilon)$ for $0<\varepsilon \leq t \leq 1$, and hence that $x(t)=x(0+)$ for $0<t \leq 1$.

This completes the proof of Lemma 3.

3. The main results. The proofs of both parts of the following theorem are based on proofs in Shohat and Tamarkin's book [13, pp. 99-101] of the case $\lambda_{n}=n$ for $n=0,1,2, \ldots$ Hildebrandt [8] originally proved this case of part (i) by a similar method.

THEOREM 1.

(i) If $M_{1}<\infty$, then there is a normalized function $\alpha \in B V$ such that (1) is satisfied and $\int_{0}^{1}|d \alpha(t)| \leq M_{1}$.

(ii) If $1<p \leq \infty$ and $M_{p}<\infty$, then there is a function $\beta \in L_{p}$ such that (2) is satisfied and $\|\beta\|_{p} \leq M_{p}$.

Proof. Define $\Lambda$ to be the linear space of functions $P$ such that

$$
P(t)=\sum_{k=0}^{m} a_{k} t^{\lambda_{k}} \text { for } 0 \leq t \leq 1,
$$

where $m$ is an arbitrary non-negative integer and $a_{0}, a_{1}, \ldots, a_{m}$ are real 
constants. Define the moment operator $\mu$ on $\Lambda$ by setting

$$
\mu(P)=\sum_{k=0}^{m} a_{k} \mu_{k}
$$

when $P$ is given by (6).

Suppose that $M_{p}<\infty$ where $1 \leq p \leq \infty$. Let $P \in \Lambda$ and let $B_{n} \in \Lambda$ be given by

$$
B_{n}(t)=\sum_{k=0}^{n} \lambda_{n k}(t) P\left(\frac{D_{k}}{D_{n}}\right) \text { for } \quad o \leq t \leq 1 .
$$

Then

$$
\mu\left(B_{n}\right)=\sum_{k=0}^{n} \lambda_{n k} P\left(\frac{D_{k}}{D_{n}}\right),
$$

and hence, by Lemma 1 ,

$$
\lim _{n \rightarrow \infty} \mu\left(B_{n}\right)=\mu(P),
$$

since, by Hölder's inequality, $M_{1} \leq M_{p}$.

Part (i). It follows from (7) that

$$
\left|\mu\left(B_{n}\right)\right| \leq M_{1}\|P\|_{C}
$$

and hence, by (8), that

$$
|\mu(P)| \leq M_{1}\|P\|_{C} .
$$

Thus $\mu$ is a bounded linear functional on a linear subspace of $C$. Hence, by the Hahn-Banach theorem [11, Theorem 5.16] and the Riesz representation theorem for bounded linear functionals on $C[2$, p. 61], there is a normalized function $\alpha \in B V$ such that, for every $P \in \Lambda$,

$$
\mu(P)=\int_{0}^{1} P(t) d \alpha(t) \text { and } \int_{0}^{1}|d \alpha(t)| \leq M_{1} .
$$

In particular, taking $P(t)=t^{\lambda_{n}}$, we get that

$$
\mu_{n}=\int_{0}^{1} t^{\lambda_{n}} d \alpha(t) \text { for } n=0,1,2, \ldots
$$

Part (ii). Let $(1 / p)+(1 / q)=1$ where $1<p \leq \infty$. Applying Hölder's inequality to (7) we get that

$$
\left|\mu\left(B_{n}\right)\right| \leq M_{p}\left(\sum_{k=0}^{n} \frac{d_{k}}{D_{n}}\left|P\left(\frac{D_{k}}{D_{n}}\right)\right|^{q}\right)^{1 / q} .
$$

Since

$$
\max _{0 \leq k \leq n} \frac{d_{k}}{D_{n}}=\max _{0 \leq k \leq n} \frac{D_{k}}{D_{n}} \frac{1}{1+\lambda_{k}} \rightarrow 0 \text { as } n \rightarrow \infty
$$


the term multiplying $M_{p}$ in the inequality tends to $\int_{0}^{1}\left(|P(t)|^{q} d t\right)^{1 / q}$. In view of $(8)$, it follows that

$$
|\mu(P)| \leq M_{p}\|P\|_{q} .
$$

Thus $\mu$ is a bounded linear functional on a linear subspace of $L_{q}$. Hence, by the Hahn-Banach theorem and the Riesz representation theorem for bounded linear functionals on $L_{q}\left[2\right.$, pp. 64, 65], there is a function $\beta \in L_{p}$ such that, for every $P \in \Lambda$,

$$
\mu(P)=\int_{0}^{1} P(t) \beta(t) d t \quad \text { and } \quad\|\beta\|_{p} \leq M_{p}
$$

In particular, taking $P(t)=t^{\lambda_{n}}$, we get that

$$
\mu_{n}=\int_{0}^{1} t^{\lambda_{n}} \beta(t) d t \text { for } n=0,1,2, \ldots
$$

This completes the proof of Theorem 1 .

Combining Lemma 2 and Theorem 1 we obtain:

\section{THEOREM 2.}

(i) $M_{1}<\infty$ if and only if (1) is satisfied by a function $\alpha \in B V$.

(ii) For $1<p \leq \infty, M_{p}<\infty$ if and only if (2) is satisfied by a function $\beta \in L_{p}$.

The next two theorems give more precise information about $M_{p}$.

\section{THEOREM 3.}

(i) If (1) is satisfied by a normalized function $\alpha \in B V$, then

$$
\begin{aligned}
& \text { (a) } M_{1}=\int_{0}^{1}|d \alpha(t)| \text { when } \lambda_{0}=0, \\
& \text { (b) } M_{1}=\int_{0}^{1}|d \alpha(t)|-|\alpha(0+)| \text { when } \lambda_{0}>0 .
\end{aligned}
$$

(ii) If $1<p \leq \infty$ and (2) is satisfied by a function $\beta \in L_{p}$, then $M_{p}=\|\beta\|_{p}$.

Proof. Part (i). By Lemma 2(i), we have that $M_{1} \leq \int_{0}^{1}|d \alpha(t)|<\infty$. Hence by Theorem 1(i), there is a normalized function $\tilde{\alpha} \in B V$ such that $\mu_{n}=\int_{0}^{1} t^{\lambda_{n}} d \tilde{\alpha}(t)$ for $n=0,1,2, \ldots$ and $\int_{0}^{1}|d \tilde{\alpha}(t)| \leq M_{1}$.

If $\lambda_{0}=0$, then, by Lemma $3, \tilde{\alpha}(t)=\alpha(t)$ for $0 \leq t \leq 1$, and hence $M_{1}=$ $\int_{0}^{1}|d \alpha(t)|$.

Suppose that $\lambda_{0}>0$, and let $\gamma(0)=0, \gamma(t)=\alpha(t)-\alpha(0+)$ for $0<t \leq 1$. Then $\mu_{n}=\int_{0}^{1} t^{\lambda_{n}} d \gamma(t)$ for $n=0,1,2, \ldots$ and hence, by Lemma 2(i), $M_{1} \leq \int_{0}^{1}|d \gamma(t)|$. Further, by Lemma 3, $\gamma(t)=\tilde{\alpha}(t)-\tilde{\alpha}(0+)$ for $0<t \leq 1$, and so, since $\gamma(0+)=$ $\gamma(0)=0$, we have that $M_{1} \leq \int_{0}^{1}|d \gamma(t)| \leq \int_{0}^{1}|d \tilde{\alpha}(t)| \leq M_{1}$. Hence $M_{1}=\int_{0}^{1}|d \gamma(t)|=$ $\int_{0}^{1}|d \alpha(t)|-|\alpha(0+)|$. 
Part (ii). By Lemma 2(ii), we have that $M_{p} \leq\|\beta\|_{p}<\infty$. Hence, by Theorem 1(ii), there is a function $\tilde{\beta} \in L_{p}$ such that $\mu_{n}=\int_{0}^{1} t^{\lambda_{n}} \tilde{\beta}(t) d t$ for $n=0,1,2, \ldots$ and $\|\tilde{\beta}\|_{p} \leq M_{p}$. By Lemma $3, \int_{0}^{t} \beta(u) d u=\int_{0}^{t} \tilde{\beta}(u) d u$ for $0 \leq t \leq 1$, and hence $\beta(t)=$ $\tilde{\beta}(t)$ a.e. in $(0,1)$. It follows that $M_{p} \leq\|\beta\|_{p}=\|\tilde{\beta}\|_{p} \leq M_{p}$, so that $M_{p}=\|\beta\|_{p}$.

This completes the proof of Theorem 3 .

THEOREM 4. If $1 \leq p \leq \infty$, then $M_{p n} \leq M_{p, n+1}$ for $n \geq 0$ and $\lim _{n \rightarrow \infty} M_{p n}=M_{p}$.

Proof. Let $0 \leq k \leq n$. Then

$$
\begin{aligned}
\lambda_{n+1, k} & =\lambda_{k+1} \cdots \lambda_{n+1} \frac{\left[\mu_{k}, \ldots, \mu_{n}\right]-\left[\mu_{k+1}, \ldots, \mu_{n+1}\right]}{\lambda_{n+1}-\lambda_{k}} \\
& =\frac{\lambda_{n+1}}{\lambda_{n+1}-\lambda_{k}} \lambda_{n k}-\frac{\lambda_{k+1}}{\lambda_{n+1}-\lambda_{k}} \lambda_{n+1, k+1}
\end{aligned}
$$

and hence

$$
\lambda_{n k}=\left(1-\frac{\lambda_{k}}{\lambda_{n+1}}\right) \lambda_{n+1, k}+\frac{\lambda_{k+1}}{\lambda_{n+1}} \lambda_{n+1, k+1}
$$

It follows that

$$
\frac{\lambda_{n k}}{d_{k}}=\left(1-\frac{\lambda_{k}}{\lambda_{n+1}}\right) \frac{\lambda_{n+1, k}}{d_{k}}+\left(\frac{1}{\lambda_{n+1}}+\frac{\lambda_{k}}{\lambda_{n+1}}\right) \frac{\lambda_{n+1, k+1}}{d_{k+1}}
$$

and hence that

$$
M_{\infty, n} \leq M_{\infty, n+1}\left(1+\frac{1}{\lambda_{n+1}}\right) \frac{D_{n}}{D_{n+1}}=M_{\infty, n+1} .
$$

Finally, for $1 \leq p<\infty$, application of Hölder's inequality to (8) yields that

$$
D_{n}^{p-1}\left|\lambda_{n k}\right|^{p} d_{k}^{1-p} \leq\left\{\left(1-\frac{\lambda_{k}}{\lambda_{n+1}}\right)\left|\lambda_{n+1, k}\right|^{p} d_{k}^{1-p}+\frac{\lambda_{k+1}}{\lambda_{n+1}}\left|\lambda_{n+1, k+1}\right|^{p} d_{k+1}^{1-p}\right\} D_{n+1}^{p-1},
$$

since

$$
1-\frac{\lambda_{k}}{\lambda_{n+1}}+\frac{\lambda_{k+1}}{\lambda_{n+1}} \frac{d_{k+1}}{d_{k}}=1+\frac{1}{\lambda_{n+1}}=\frac{D_{n+1}}{D_{n}} .
$$

Summing the above inequality for $k=0,1, \ldots, n$, we get that

$$
M_{p n}^{p} \leq M_{p, n+1}^{p}-\frac{\lambda_{0}}{\lambda_{n+1}}\left|\lambda_{n+1,0}\right|^{p} d_{0}^{1-p} D_{n+1}^{p-1} \leq M_{p, n+1}^{p}
$$

This completes the proof of Theorem 4 .

\section{REFERENCES}

1. N. I. Akhiezer. The Classical Moment Problem, Oliver and Boyd, Edinburgh, 1965.

2. S. Banach, Théories des Opérations Linéaires, Warsaw, 1932. 
3. D. L. Berman, Application of interpolatory polynomial operators to solve the moment problem, Ukrain Math. Ž. 14 (1963), 184-190.

4. K. Endl, On systems of linear inequalities in infinitely many variables and generalized Hausdorff means, Math. Z. 82 (1963), 1-7.

5. F. Hausdorff, Summationsmethoden und Momentfolgen I, Math. Z. 9 (1921), 74-109.

6. F. Hausdorff, Summationsmethoden und Momentfolgen II, Math. Z. 9 (1921), 280-299.

7. F. Hausdorff, Momentprobleme für ein eindliches Interval, Math. Z. 16 (1923), 220-248.

8. T. H. Hildebrandt, On the moment problem for a finite interval, Bull. A.M.S. 38 (1932), 269-270.

9. D. Leviatan, A generalized moment problem, Israel J. Math. 5 (1967), 97-103.

10. D. Leviatan, Some moment problems in a finite interval, Can. J. Math. 20 (1968), 960-966.

11. W. Rudin, Real and Complex Analysis, Second edition, McGraw-Hill, 1974.

12. I. J. Schoenberg, On finite rowed systems of linear inequalities in infinitely many variables, Trans. A.M.S. 34 (1932) 594-619.

13. J. A. Shohat and J. D. Tamarkin, The Problem of Moments, A.M.S., 1943.

14. D. V. Widder, The Laplace Transform, Princeton, 1946.

DepartMent OF MATHEMATICS

THE UNIVERSITY OF WESTERN ONTARIO

London, Ontario, Canada n6a 5B9 